\title{
Detailed semiautomated MRI based morphometry of the neonatal brain: Preliminary results
}

\author{
Mitsuhiro Nishida, ${ }^{\text {a,b }}$ Nikolaos Makris, ${ }^{\text {a }}$ David N. Kennedy, ${ }^{\text {a }}$ Mark Vangel, ${ }^{\text {c }}$ Bruce Fischl, \\ Kalpathy S. Krishnamoorthy, ${ }^{\mathrm{d}}$ Verne S. Caviness, ${ }^{\mathrm{d}}$ and P. Ellen Grant ${ }^{\mathrm{c}, \mathrm{e}, *}$ \\ ${ }^{a}$ Center for Morphometric Analysis, Department of Neurology, Massachusetts General Hospital, MA 02114, USA \\ ${ }^{\mathrm{b}}$ Department of Pediatrics, School of Medicine, Keio University, Tokyo, Japan \\ 'Department of Radiology, The Athinoula A. Martinos Center for Biomedical Imaging, Massachusetts General Hospital, MA 02114, USA \\ ${ }^{\mathrm{d}}$ Department of Neurology, Division of Pediatric Neurology, Massachusetts General Hospital, MA 02114, USA \\ ${ }^{\mathrm{e}}$ Department of Radiology, Division of Pediatric Radiology, Massachusetts General Hospital, 34 Fruit Street, Ellison 237, Boston, MA 02114, USA
}

Received 22 November 2005; revised 13 March 2006; accepted 3 May 2006

Available online 20 July 2006

In the neonate, regional growth trajectories provide information about the coordinated development of cerebral substructures and help identify regional vulnerability by identifying times of faster growth. Segmentation of magnetic resonance images (MRI) has provided detailed information for the myelinated brain but few reports of regional neonatal brain growth exist. We report the method and preliminary results of detailed semiautomated segmentation of 12 normative neonatal brains (gestational age 31.1-42.6 weeks at time of MRI) using volumetric T1-weighted images. Accuracy was confirmed by expert review of every segmented image. In 5 brains, repeat segmentation resulted in intraclass correlation coefficients $>0.9$ (except for the right amygdala) and an average percent voxel overlap of $90.0 \%$. Artifacts or image quality limited the number of regions segmented in 9/12 data sets and 1/12 was excluded from volumetric analysis due to ventriculomegaly. Brains were segmented into cerebral exterior $(N=8)$, cerebral lobes $(N=5)$, lateral ventricles $(N=8)$, cerebral cortex $(N=6)$, white matter $(N=6)$, corpus callosum $(N=7)$, deep central gray $(N=8)$, hippocampi $(N=8)$, amygdalae $(N=8)$, cerebellar hemispheres $(N=8)$, vermis $(N=8)$, midbrain $(N=8)$, pons $(N=8)$ and medulla $(N=8)$. Linear growth $(P<0.05)$ was identified in all regions except the cerebral white matter, medulla and ventricles. Striking differences in regional growth rates were noted. These preliminary results are consistent with the heterochronous nature of cerebral development and provide initial estimates of regional brain growth and therefore regional vulnerability in the perinatal time period.

(c) 2006 Elsevier Inc. All rights reserved.

Keywords: Morphometry; Brain; Development; Neonate; MRI; Gompertz

\footnotetext{
* Corresponding author. Department of Radiology, Division of Pediatric Radiology, Massachusetts General Hospital, 34 Fruit Street, Ellison 237, Boston, MA 02114, USA. Fax: +1 6177268360 .

E-mail address: ellen@nmr.mgh.harvard.edu (P.E. Grant).

Available online on ScienceDirect (www.sciencedirect.com).
}

\section{Introduction}

The size and form of the human brain are the result of a complex series of overlapping histogenetic processes influenced by genes, environmental exposures and epigenetic rules (heritable modifications of DNA bases that can lead to rapidly reversible changes in gene expression). Although cerebral development begins in the early embryonic period and continues through the second decade of postnatal life (Sidman and Rakic, 1982), it is during the period of rapid growth that the brain is most vulnerable to errors arising from genetic defects, environmental exposures and abnormal gene expression. This vulnerable period of rapid growth occurs between the third trimester of pregnancy and the early postnatal months. During this phase, the brain weight increases by $6 \%$ of adult weight/ month, and there are associated radical changes in shape and surface features (Caviness et al., 1996a,b,c). The rate of growth decelerates by a factor of 2 by 10 months and by a factor of 4 by 18 months. The asymptote of brain growth, approximately $80 \%$, is reached by the third year of life, with the brain increasing only some $8-10 \%$ in volume beyond the 9th year of life (Caviness et al., 1996a,b,c). During the phase of rapid growth, there is also rapid development of axono-dendritic connections and myelination followed by neuronal specification which results in programmed cell death and pruning of axonal and dendritic arbors (Bourgeois and Rakic, 1993; LaMantia and Rakic, 1990, 1994). Therefore, not only is the brain most vulnerable from the third trimester to the early postnatal months, but also insults occurring during this time period have profound effects on the orchestration of neuronal connectivity and the integration of neural activity.

It has been estimated that any process impairing brain growth such that the head circumference is not normal by 8 months will result in life long disabilities in the cognitive if not other domains of cerebral function (Hack and Breslau, 1986; Hack et al., 1989, 1991). Recent MRI studies in patients with ADHD have detected abnormal caudate volumes prior to adolescence and focal changes in white 
matter and gray matter volumes (Castellanos et al., 2002; Filipek et al., 1997; Spencer et al., 2002; Wilens et al., 2002), suggesting that regional volumetric differences on MRI are associated with variations in cerebral function. There is also evidence based on MRI morphometry that volumetric changes precede clinical expression in certain grave neuropsychiatric disorders. In particular, volumetric changes in schizophrenia appear to have predictive power for eventual clinical expression among first degree family members of probands (Pantelis et al., 2003). Therefore, it follows that the pace of regional brain growth as measured with MRI may be a sensitive indicator of the biological state of the brain and may provide predictions of those neonates and young children at risk for abnormal cerebral function. Volumetric measures based upon MRI in the first weeks and months of life may thus be a more sensitive index of future neurological outcome than formal behavioral testing and neurological examination as these latter indices are notoriously insensitive as predictors of neurological outcome.

Almost all automated and semiautomated MRI segmentation techniques for cerebral morphometry have been developed for volumetric T1 studies of the fully myelinated mature brain (Filipek et al., 1994; Fischl et al., 2002; Gogtay et al., 2004; Sowell et al., 2004). The most comprehensive and systematic but labor intensive approach is the detailed computer-assisted semiautomated technique developed by the Center for Morphometric Analysis (Caviness et al., 1989; Filipek et al., 1989, 1991, 1992, 1994; Kennedy, 1986; Kennedy et al., 1989; Kennedy and Nelson, 1987). In this technique, segmentation is partially automated on a slice by slice basis with the investigator interacting with the segmentation process and guiding the segmentation with judgments that are knowledge based. This technique assesses reliability in the sense of percent voxel concordance among operators knowledgeable with regard to anatomy. This detailed semiautomated approach is highly accurate because an experienced reader guides and confirms accurate segmentation on a slice by slice basis but is very costly in terms of time required of experienced analysts. As a result, this computer-assisted detailed semiautomated technique does not allow the rapid and less expensive evaluation of large numbers of MRI studies that automated and semiautomated techniques allow. Instead, this approach allows the most accurate evaluation of a small number of MR studies and the creation of a set of "gold standards" which can be used to develop and validate more rapid and practical semiautomated and automated techniques.

Analysis techniques developed for the mature brain are not directly transferable to the incompletely myelinated brain. This is particularly true for the newborn brain where the gray-white matter contrast is inverted with white matter lower in T1 signal than cortex. In addition, the regional variation in myelin maturation results in much more regional variation in both gray and white matter T1 signals compared to adults. Further difficulty is added by the smaller head sizes, lack of myelin, and need for shorter scan times to avoid motion which result in T1 images with lower signal to noise ratios. Where semiautomated techniques for the immature brain have been described, these have provided useful approximates of the volumes of major brain regions (Huppi et al., 1998; Peterson et al., 2003) and have been useful in searching for differences between two groups of patients (Huppi et al., 1998; Inder et al., 1999; Murphy et al., 2001). However, these methods do not include slice by slice validation by an experienced reviewer and therefore although reproducible may not be accurate in their volume estimates of specific brain structures, in particularly those that are relatively small.
The purpose of this study was to develop a knowledge based, reliable segmentation process for newborn brains based on the methods previously developed for adult brains at the Center for Morphometric Analysis, of the Massachusetts General Hospital. This paper will describe our new in vivo MR-based technique for segmentation of the minimally myelinated neonatal brain and the results obtained from segmenting normative neonatal brains at various gestational ages.

\section{Materials and methods}

Subjects

A total of 12 neonates, 6 female and 6 male, with gestational ages at birth ranging from 31.1 to 42.6 weeks, imaged between 1 and 39 days of life were included in this study. All were imaged for clinical indications, and the study was approved by our institutional review board. Clinical indications typically included evaluation of suspected seizures, perinatal depression, suspected hemorrhage, or questionable ultrasound findings. On expert review by a pediatric neuroradiologist (PEG), the MR images showed no structural abnormalities apart from one case with mildly enlarged ventricles. This case was included in the reliability measures for the segmentation procedure but was excluded from the normative data analysis. None of the studies had diffusion-weighted imaging (DWI) abnormalities, apart from a small thalamic stroke in two patients, none had MR spectroscopy abnormalities and none had hemorrhage. Only one patient required mechanical ventilation for respiratory distress for more than $24 \mathrm{~h}$. None met the clinical criteria for hypoxic-ischemic encephalopathy, and none were septic or had persistent hypoglycemia. There was no imaging or clinical evidence of congenital malformations or metabolic disorders. None had abnormal neurological exams. Three of four neonates with confirmed seizures had normal EEGs and normal neurological exams at follow-up evaluation. The remaining case had an improving EEG with the expectation that seizure medications would be terminated by 18 months of age. Three with suspected neonatal seizures had no further events suggestive of seizures.

\section{MR image acquisition}

An axial 3D SPGR (volumetric spoiled gradient echo) sequence was performed as part of the clinical protocol on a 1.5-T MR System (General Electric, Milwaukee). This is a T1-weighted image with imaging parameters as follows: TR/TE $=30 / 8$, flip angle $=25$ to 30 , matrix $=256 \times 192$, bandwidth 10.42 , slice thickness $=1.2$ to $1.4 \mathrm{~mm}, \mathrm{FOV}=220 \times 165 \mathrm{~mm}$ or $200 \times 150$ $\mathrm{mm}, \mathrm{NEX}=1$. Therefore, spatial resolution was between 0.8 and $0.9 \mathrm{~mm} \times 0.8$ to $0.9 \mathrm{~mm} \times 1.2 \mathrm{~mm}$. For five neonates, imaging was performed with a Pediatric Head Coil (Scan Med) but when possible $(N=7)$ imaging was performed with a Neonatal Head Coil (Advanced Imaging Research) due to the marked improvement in SNR with the smaller neonate-specific head coil. Both coils are receive only coils and therefore do not result in different image distortions.

\section{Positional normalization}

To reduce variability due to differences in head position, all brains are repositioned in three dimensions using a reference plane that bisects the decussations of the anterior commissure (AC) and posterior 
commissure (PC), and the interhemispheric fissure at the level of the PC in the coronal plane. After repositioning, a tri-linear interpolation method was used to resample the MR image data set to a resolution of $1.0-1.4 \mathrm{~mm}$ in the newly defined, "normalized" coronal plane and $0.4-0.625 \mathrm{~mm}$ in the sagittal and axial planes for optimal visualization and subsequent segmentation (Filipek et al., 1991).

\section{Image segmentation}

At the Center for Morphometric Analysis (CMA), a technique for reliably and reproducibly segmenting volumetric T1-weighted images of mature, fully myelinated brains has been developed and extensively published (Caviness et al., 1996a,b,c; Kennedy et al., 1994; Makris et al., 1999; Meyer et al., 1999; Rademacher et al., 1992). The segmenting process includes intensity contour mapping and histogram analysis of the signal intensity distributions (Kennedy et al., 1989). Intensity contour mapping is the process of manually selecting an intensity threshold that results in a continuous isointense outline to define a specific anatomic border. Manual editing, as anatomically necessary, is made to complete the anatomic border. These manually guided boundaries are created in the coronal plane with placement guided by detailed knowledge of the regional anatomy, reformations in the sagittal and coronal plane and boundaries drawn on the sagittal and axial plane projected into the coronal plane. When a histogram analysis of signal intensity distributions is used to define the boundary between two regions, the operator manually defines a small region that encompasses these two regions. If the signal intensity characteristics of the manually selected region are a histogram with a bimodal distribution, the nadir defines the border between the two regions. When the boundary is defined by histogram analysis, the images are still further edited on a slice by slice basis using intensity contour mapping guided by detailed anatomical knowledge. If a bimodal distribution of signal intensities is not obtained, the histogram method is abandoned and intensity contour mapping guided by detailed anatomical knowledge is used. Further details of these operations are described elsewhere (Caviness et al., 1989; Filipek et al., 1989, 1991, 1992; Kennedy, 1986; Kennedy et al., 1989; Kennedy and Nelson, 1987).

Using a combination of these techniques, the "mature brain" brain (age greater than 4 years) is separated from the overlying structures and divided into forebrain, brainstem, and cerebellum. The forebrain is further segmented into cerebral cortex, cerebral white matter, corpus callosum, deep central gray (caudate nuclei, lentiform nuclei, thalami, and intervening white matter), hippocampi, and amygdalae (Fig. 1). The brainstem is further segmented into midbrain, pons and medulla and the cerebellum, into vermis and cerebellar hemispheres. The details of the anatomical boundaries used to segment these regions are described elsewhere (Caviness et al., 1989; Filipek et al., 1989, 1991, 1992; Kennedy, 1986; Kennedy et al., 1989; Kennedy and Nelson, 1987).

We have refined the anatomic segmentation methodology applied to the mature brain for segmentation of the immature brain. Accurate segmentation of the neonatal brain using landmarks defined in the adult technique required the neuroradiologist teach the segmenter the different appearance of neonatal brain structures on T1-weighted images. When the segmentation technique for mature brains did not yield anatomically accurate segmentation by visual inspection by an experienced pediatric neuroradiologist (PEG), the technique was modified to achieve the most accurate segmentation based on knowledge of the underlying anatomy. A total of three major modifications were required. The first modification required segmenting the caudate nuclei, lentiform nuclei and thalami en bloc as there was insufficient contrast to accurately segment these structures individually. The second modification was to replace histogram-based segmentation techniques by intensity contour mapping for segmentation of the graywhite matter junction. In the neonate low signal to noise results in histograms that are not bimodal or there are regional variations in signal due to regional variations in myelin which yield results that are only accurate over a small region. The third modification required defining the cavum septum pellucidum as a separate structure and therefore not including it in either the ventricular or brain volumes. In a subset of five cases, the cortex was further segmented into right and left frontal, temporal, parietal, occipital and limbic lobes. Landmarks to subdivide the cortices were adapted from the previously published techniques (Caviness et al., 1996a,b,c; Rademacher et al., 1992, 1993). Lobar parcellation is possible using this technique from approximately 30 weeks gestational age. After the neonatal technique was established, each segmented brain was checked by the neuroradiologist for consistency. At this point, either no or only minimal corrections were necessary.

Artifacts, poor quality images or incomplete coverage resulted in the exclusion of some data sets. Of the 11 studies judged to have normal imaging findings, $6 / 11$ were used to define whole brain volumes, 8/11 were used for cerebral exterior measurements, 6/11 for cerebral cortex and white matter, 7/11 for corpus callosum, 8/11 for the remaining supratentorial structures, and $8 / 11$ for posterior fossa segmentation. Overall it took up approximately 7 days to segment one brain into all 30 regions. Defining the cortical surface and gray-white junction required the longest time, 3 to 5 days, and the smaller remaining regions between 1 and 2 days. Lobar segmentation required only 3 to $4 \mathrm{~h}$.

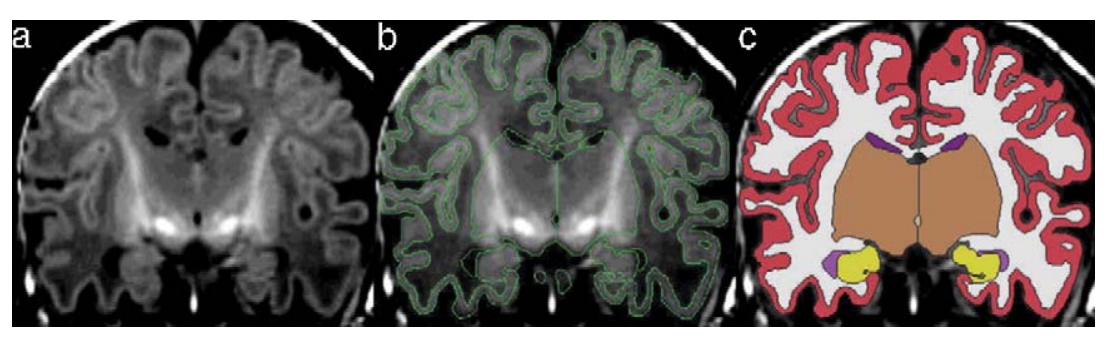

Fig. 1. Knowledge-based detailed semiautomated segmentation. (a) Coronal reformation of axially acquired 3D SPGR data after positional normalization. (b) Results of detailed semiautomated segmentation (green lines). (c) Color-coded segmentation with cortex (red), white matter (white), deep central gray (brown), hippocampi (yellow) and ventricular CSF (purple) shown here. (For interpretation of the references to colour in this figure legend, the reader is referred to the web version of this article.) 


\section{Intrarater reliability}

A single operator $(\mathrm{MN})$ segmented the 36 brain regions twice in at least 5 subjects to allow estimation of intrarater reliability. The technician was blind to the concordance of cases across repetitions. To determine reliability, we calculated intraclass correlation coefficients (ICC) for the absolute agreement (McGraw and Wong, 1996; Shrout and Fleiss, 1979) of the two volume measurements. In addition we calculated percent overlap of voxels in the two volume measurements (percent common voxels, PCV). All brain regions had an ICC greater than 0.90 with the exception of the right amygdala (ICC $=>0.84, P=0.22$ ). The percent overlap exceeded $80 \%$ with an average percent overlap of $90.0 \%$. No consistent variation of reliability as a function of gestational age was found. The percent overlap of volumes was slightly better for studies obtained with the Neonatal Head Coil (Advanced Imaging Research) compared to those obtained with the Pediatric Head Coil (Scan Med) (90.5\% compared to $88.8 \%$ ) even though they had the same mean corrected gestational age of 37.5 weeks.

\section{Volumetric analysis}

As in the technique for the mature brain, the number of voxels in each unit on the coronal image was multiplied by the volume per voxel. The volume obtained for each unit is summated for all slices in which each unit appears (Kennedy et al., 1989; Kennedy and Nelson, 1987). Whole brain volumes include cerebrum, cerebellum, brainstem, and midbrain with ventricles excluded. Cerebral exterior includes cerebrum, lateral and inferior lateral ventricles but excludes the 3rd ventricle and cavum septi pellucidi. Left- and right-sided volumes were calculated independently and added together to give the total volume. The total volume of segmented structures was then plotted as a function of corrected gestational age. Linear regressions were preformed, and a $t$ test was used to assess the probability that the volume of each structure versus corrected gestational age was linear with $P$ values less than 0.05 considered significant.

\section{Surface rendering}

Since topological changes are as important as volumetric changes, each segmented volume was displayed as a twodimensional surface in three-dimensional space using $\mathrm{SV}^{2}$ [Sean McInerney, Center for Morphometric Analysis]. This program creates surface reconstructions of the segmented volumes allowing

$\overline{\text { Fig. 2. Regional cerebral growth trajectories. (a) Total cerebral white matter }}$ (CWM) and deep central gray (DCG) volumes versus corrected gestational age (GA) at time of MRI scan. White matter volumes did not increase linearly but leveled off after approximately 37 weeks cGA. Total DCG volumes increased linearly $(P<0.05)$ with the best fit $y=1.48 x-35.24$ $\left(r^{2}=0.98\right)$. (b) Total hippocampi, amygdalae and corpus callosum volumes versus corrected GA at time of MRI scan. All volumes increased linearly $(P<0.05)$ with best fits $y=0.08 x-1.08\left(r^{2}=0.90\right)$ for the corpus callosum, $y=0.09 x-1.82\left(r^{2}=0.91\right)$ for the hippocampus and $y=$ $0.06 x-1.46\left(r^{2}=0.64\right)$ for the amygdalae. The linear fit for the population model of total hippocampal volumes is shown for comparison. (c) Total cerebral gray matter (CGM) and cerebral white (CWM) volumes as a fraction of whole brain volume versus corrected GA. CGM fraction increased linearly $(P<0.05)$ with best fit $y=0.02 x-0.16\left(r^{2}=1.00\right)$. CWM fraction decreased linearly $(P<0.05)$ with best fit $y=0.02 x+1.17$ $\left(r^{2}=0.99\right)$. (Note that there are 5 subjects in this graph as one subject with whole brain volumes did not have gray and white matter volumes defined). qualitative assessment of topological changes. Visualization of surfaces created from segmented regions also allows a visual assessment of the segmentation quality.

\section{Results}

Volumetric analysis

Results of volumetric analysis are show in Figs. 2-5. All regions showed significant linear increases in volume with
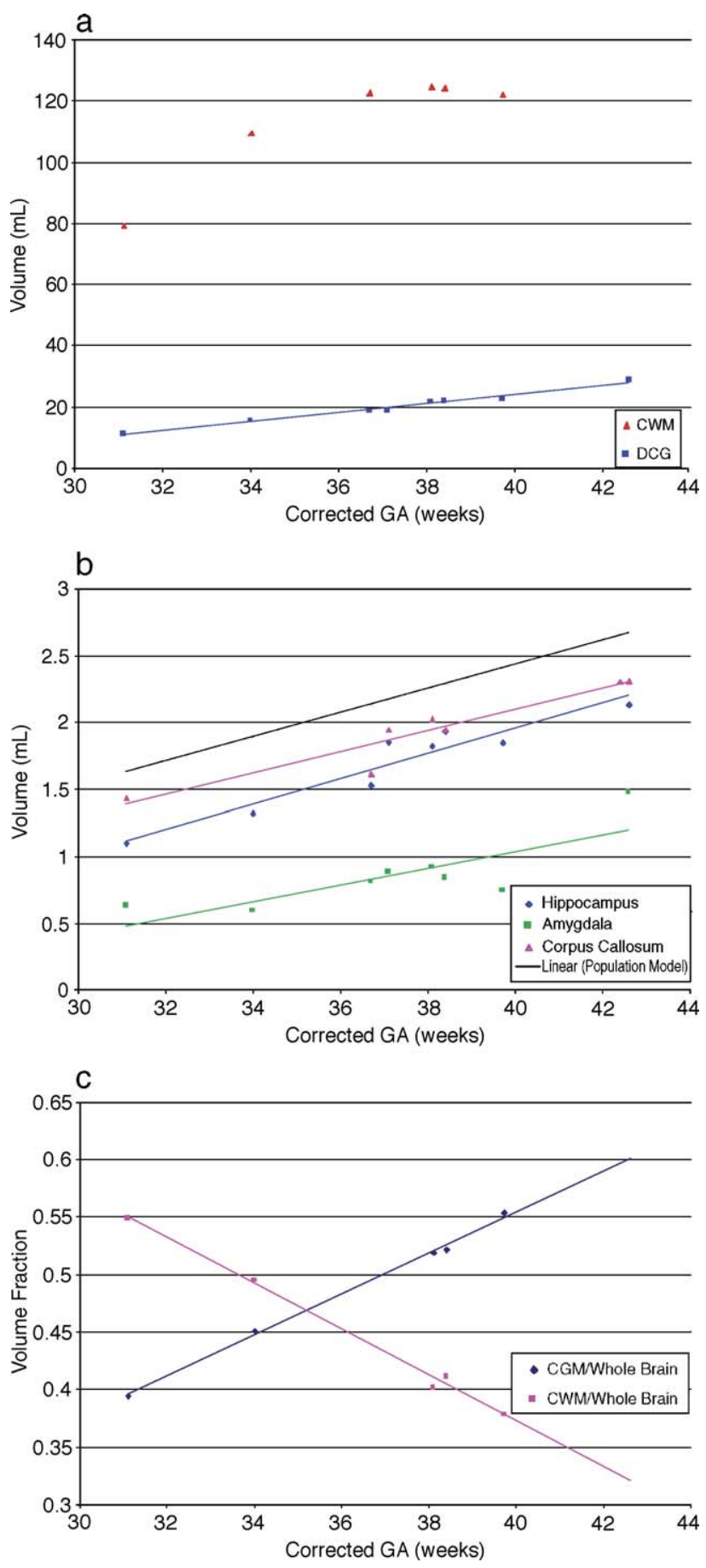

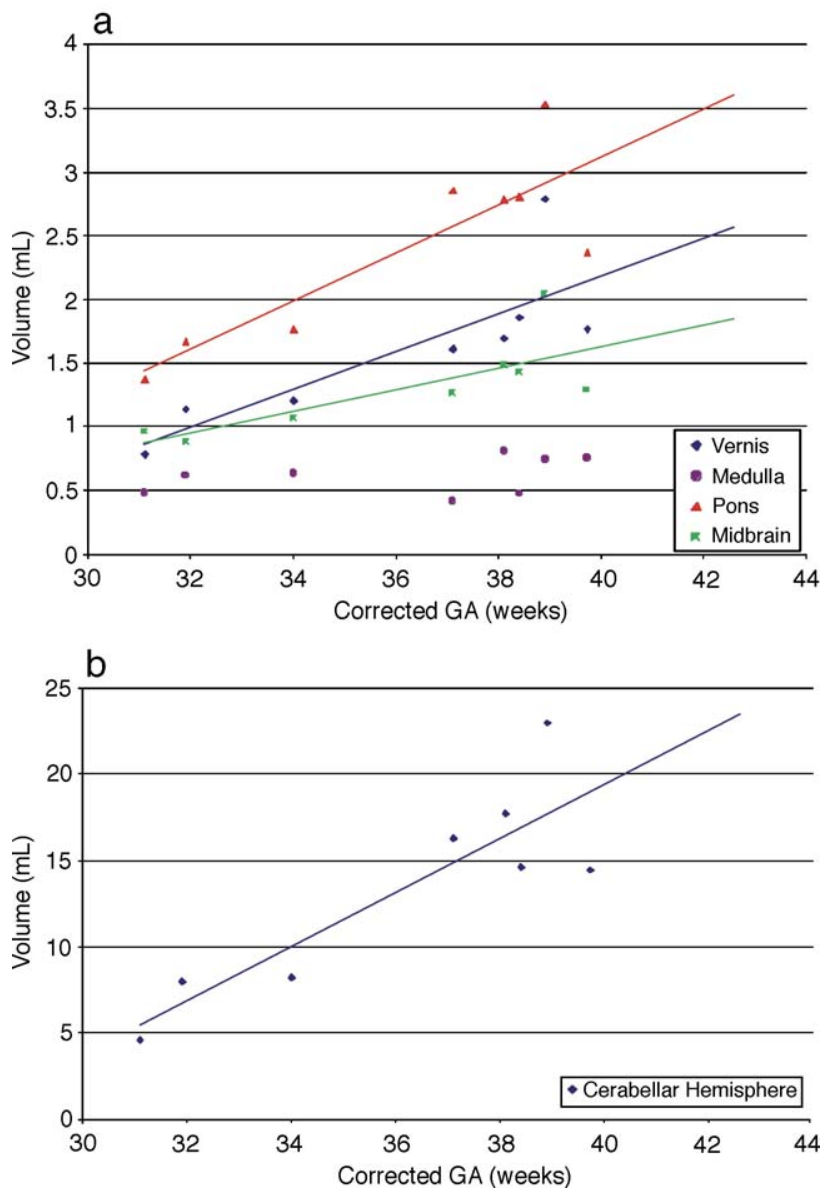

Fig. 3. Regional posterior fossa growth trajectories. (a) Vermis, medulla, pons, and midbrain volumes versus corrected GA at time of MRI scan. All volumes increased linearly $(P<0.05)$ with the exception of the medulla. The best fits were $y=0.19 x-4.43\left(r^{2}=0.73\right)$ for the pons, $y=0.15 x-$ $3.75\left(r^{2}=0.67\right)$ for the vermis and $y=0.08 x-1.75\left(r^{2}=0.59\right)$ for the midbrain. (b) Total cerebellar hemisphere volumes versus corrected GA at time of MRI scan. The volume increased linearly $(P<0.05)$ with best fit $y=1.56 x-43.16\left(r^{2}=0.75\right)$.

gestational age at the time of the scan $(P<0.05)$ with the exception of the cerebral white matter, ventricles, and medulla. There were no significant differences in volumes of the left and right brain structures, and therefore, these were combined. Striking differences in regional brain growth were identified. The whole brain (without ventricles) had a growth rate of $21.36 \mathrm{ml} /$ week $\left(R^{2}=\right.$ $0.96)$, with the cerebral cortical gray increasing at the most rapid rate of $12.57 \mathrm{ml} /$ week $\left(R^{2}=0.99\right)$. As expected, much slower growth rates were noted in smaller structures such as the deep central gray $\left(1.5 \mathrm{ml} /\right.$ week, $\left.R^{2}=0.98\right)$, hippocampi $(0.09 \mathrm{ml} /$ week, $\left.R^{2}=0.91\right)$ and amygdalae $\left(0.06 \mathrm{ml} /\right.$ week, $\left.R^{2}=64\right)$. Although the corpus callosum volume increased linearly at a rate of $0.08 \mathrm{ml} /$ week $\left(R^{2}=0.90\right)$, overall the cerebral white matter volume appeared to level off after approximately 36 weeks. The ventricles and medulla showed no detectable growth. When expressed as a percent of the cerebrum, gray matter showed a linear increase in volume with corrected gestation age $\left(1.2 \%\right.$ per week, $\left.R^{2}=1.00\right)$, whereas the white matter showed a linear decrease $(-2 \%$ per week, $\left.R^{2}=0.99\right)$. When segmented into lobes, the fastest growth rate was in the frontal lobes at $4.44 \mathrm{ml} /$ week $\left(R^{2}=0.97\right)$ followed by the parietal lobes at $2.93 \mathrm{ml} /$ week $\left(R^{2}=0.99\right)$, temporal lobes at 2.29 $\mathrm{ml} /$ week $\left(R^{2}=0.96\right)$, occipital lobes at $2.40 \mathrm{ml} /$ week and finally limbic lobes at $0.58 \mathrm{ml} /$ week $\left(R^{2}=0.96\right)$. In the posterior fossa, the cerebellar hemispheres showed a much faster growth rate $(1.7 \mathrm{ml} /$ week, $\left.R^{2}=0.77\right)$ than the pons $\left(0.19 \mathrm{ml} /\right.$ week, $\left.R^{2}=0.73\right)$ and vermis $\left(0.14 \mathrm{ml} /\right.$ week, $\left.R^{2}=0.70\right)$.

\section{Surface rendering}

The surface rendered images of the cerebral exterior showed clear progression of gyral complexity (Fig. 6a). It is interesting to note that the downward angulation of the temporal lobe appears to develop between 31.1 and 34 weeks. The gyri and sulci are less apparent on the 39.7 week brain as the gyri were apposed with no


Fig. 4. Growth trajectories and comparisons to prior studies. (a) Whole brain with ventricles volumes in $\mathrm{ml}$ versus corrected GA at time of scan in weeks. Our data (red dots) with $95 \%$ confidence band. The volume increased linearly $(P<0.05)$ with best fit $y=21.57 x-514.31\left(r^{2}=0.96\right)$. Volumes from the Gompertz population model using fresh specimens (McLennan et al., 1983) is within our 95\% confidence band. Volumes from the Gompertz population model using fixed specimens (Koop et al., 1986) is shifted to larger volumes outside our $95 \%$ confidence band. (b) Whole brain volumes in milliliters versus corrected GA at time of scan in weeks. Our data (black dots) with $95 \%$ confidence band. The volume increased linearly $(P<0.05)$ with best fit $y=21.39 x-511.08\left(r^{2}=0.96\right)$. Volumes generated from the Huppi population model using MRI data (Huppi et al., 1998 ) is shifted to larger volumes outside our $95 \%$ confidence band. 
a

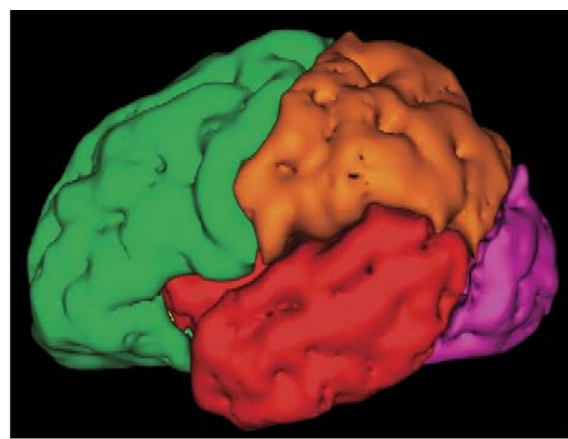

b

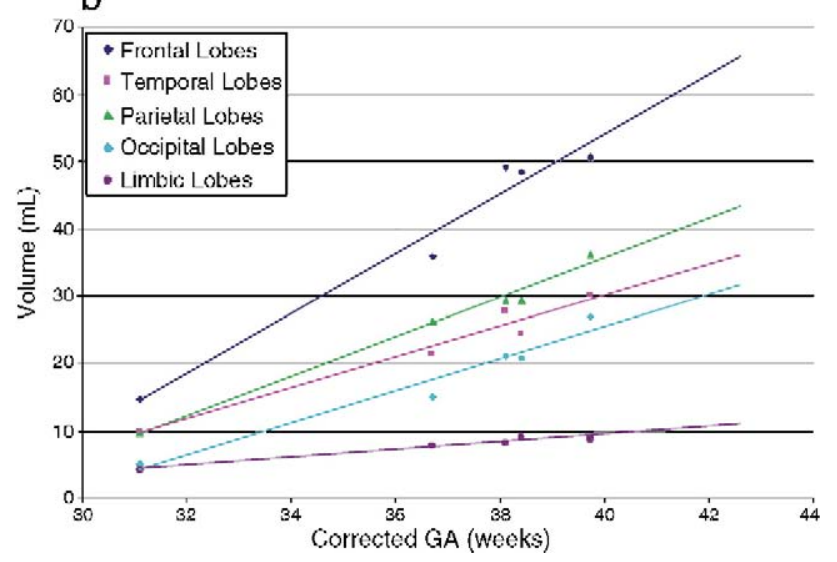

Fig. 5. Lobar growth trajectories. (a) Lateral view of the color coded lobar segmentation showing frontal (green), parietal (orange), temporal (red) and occipital (purple) lobes. (b) Frontal, temporal, parietal, occipital and limbic lobe growth versus corrected GA at time of MRI scan. All volumes increased linearly $(P<0.05)$ with best fits $y=4.44 x-123.40\left(r^{2}=0.97\right)$ for the frontal lobes, $y=2.93 x-81.72\left(r^{2}=0.99\right)$ for the parietal lobes, $y=2.29 x-61.51$ $\left(r^{2}=0.96\right)$ for the temporal lobes, $y=2.40 x-70.35\left(r^{2}=0.95\right)$ for the occipital lobes, and $y=0.58 x-13.53\left(r^{2}=0.96\right)$ for the limbic lobes. (For interpretation of the references to colour in this figure legend, the reader is referred to the web version of this article.)

discernable CSF in the sulci to aid segmentation. On the surface rendered images of the cerebellum and vermis, the faster growth rate of the cerebellar hemispheres compared to the vermis is easily appreciated (Fig. 6b). With increasing gestational age, the cerebellar hemispheres grow along their long axis (length) but appear to increase in width more than in length and increasingly surrounding the vermis. Although in both Figs. $6 \mathrm{a}$ and $\mathrm{b}$ a clear trend to increased volume can be seen, there is some variation that could either be noise, biological variability or the inadvertent inclusion of subjects that may develop disorders later in life. Larger prospective studies of normal newborns with neuropsychiatric evaluations after at least 5 years of age to confirm their normal cognitive function will be needed to understand the contribution of these different factors.

\section{Discussion}

This paper describes a detailed semiautomated segmentation method that reliably and reproducibly segments minimally myelinated neonatal brains into cerebral exterior, cerebral lobes, lateral ventricles, cerebral cortex, cerebral white matter, corpus callosum, deep central gray, hippocampi, amygdalae, cerebellar hemispheres, vermis, midbrain, pons and medulla. To our knowledge, this is the first detailed semiautomated segmentation method and the most detailed segmentation procedure for neonatal brains to date. In this cross-sectional study, all regions, except the cerebral white matter, medulla and ventricles, showed linear growth from corrected gestational age of 31.1 to 42.6 weeks. Striking regional differences in growth rates were identified. During this period, gray matter increased from $40 \%$ to just over $55 \%$ of the total brain volume while white matter decreased from approximately $50 \%$ to just under $38 \%$ of the total brain volume. These results confirm the heterochronus nature of cerebral growth and provide initial estimates of regional brain growth and therefore regional vulnerability in the perinatal time period.

Further insight into the significance of these growth curves can be obtained when the data is normalized for size by expressing size as a fraction of previously published adult volumes. The fractional volumes obtained provide an estimate of the size of each structure relative to its target adult volume. Fig. 7 shows fractional growth of structures that had comparable adult volumes previously calculated at the Center for Morphometric Analysis with our established adult technique (Filipek et al., 1994). Each structure starts out at a different fraction of its adult volume and show different rates of fractional growth. The deep central gray nuclei start out with the highest fractional volume and has the steepest slope and therefore most rapid rise in fractional volume. This suggests that the deep central gray nuclei take the lead in cerebral development at this stage. The minimal white matter growth in the presence of rapid gray matter growth implies that cerebral white matter is essentially in abeyance until axonal pathways are established.

The largest study of normative brain growth in the neonatal time period comes from specimens obtained postmortem and is based on data from the Collaborative Perinatal Project of the National Institute of Neurological and Communicative Disorders and Stroke (NINCDS), reported by McLennan et al. (1983). Using over 1233 fresh fetal and neonatal brains, the Gompertz function, a model of self-limited growth, was found to provide the best fit for brain growth by weight up to 44 weeks gestational age using the following parameters:

$y=1065 * \exp [-\exp (2.108-0.04981 x)]$

where $y$ is the brain weight in grams, and $x$ is the gestational age in weeks. For gestational ages between 31 and 43 weeks evaluated here, this curve is essentially linear. Therefore, assuming specific gravity remains constant at $1.032 \mathrm{~g} / \mathrm{ml}$ (Kretschmann et al., 1986a,b), a linear growth rate in cerebral volume is also expected in the age range we studied. The volumetric estimates from this study result in cerebral volumes that are well within the $95 \%$ confidence interval for our results (Fig. 4a).

Using 24 fixed brain volumes, Koop et al. (1986) calculated a slightly different Gompertz function for brain growth by volume,

$y=1061 * \exp [-\exp (2.16-0.0049 x)]$

where $y$ is the entire brain volume in milliliters, and $x$ is the GA in weeks. This curve is also essentially linear in the range of GA we studied. These volumetric estimates are on average $41 \%$ larger than our whole brain volumes plus lateral ventricles we measured and are outside our confidence interval (Fig. 4a). His rhombencephalon plus cerebellum values are also $44 \% \pm$ $28 \%$ larger than our calculations (Kretschmann et al., 1986a,b). 
a

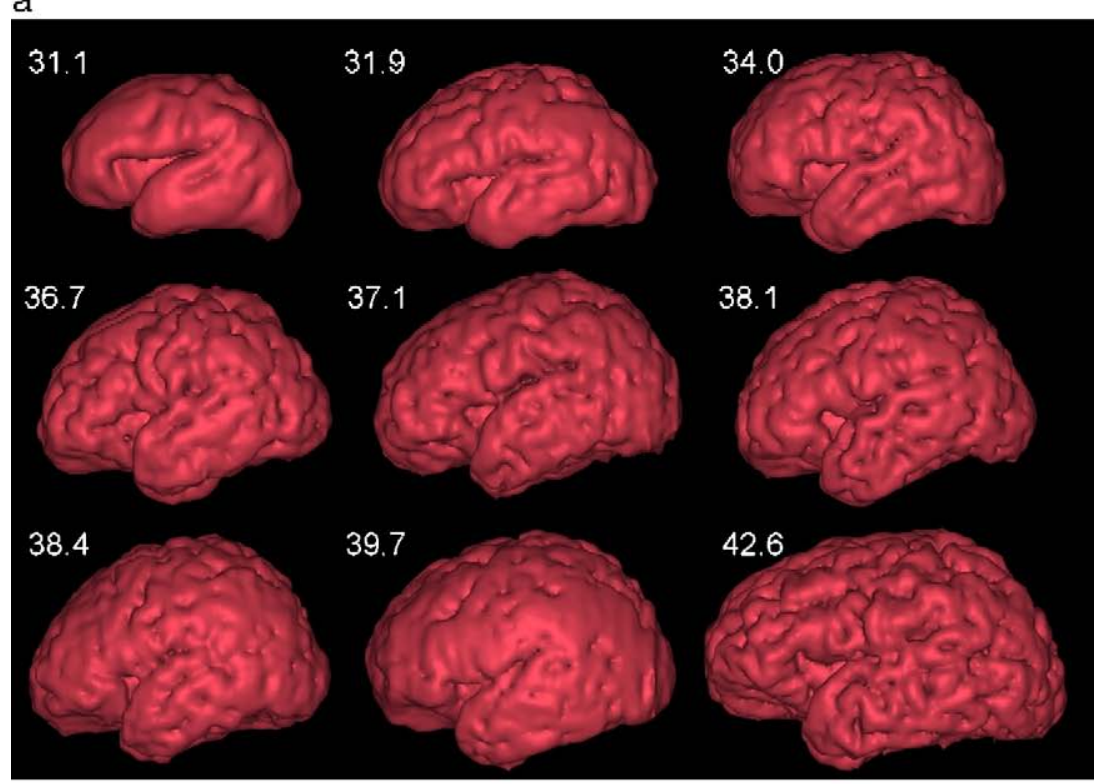

b

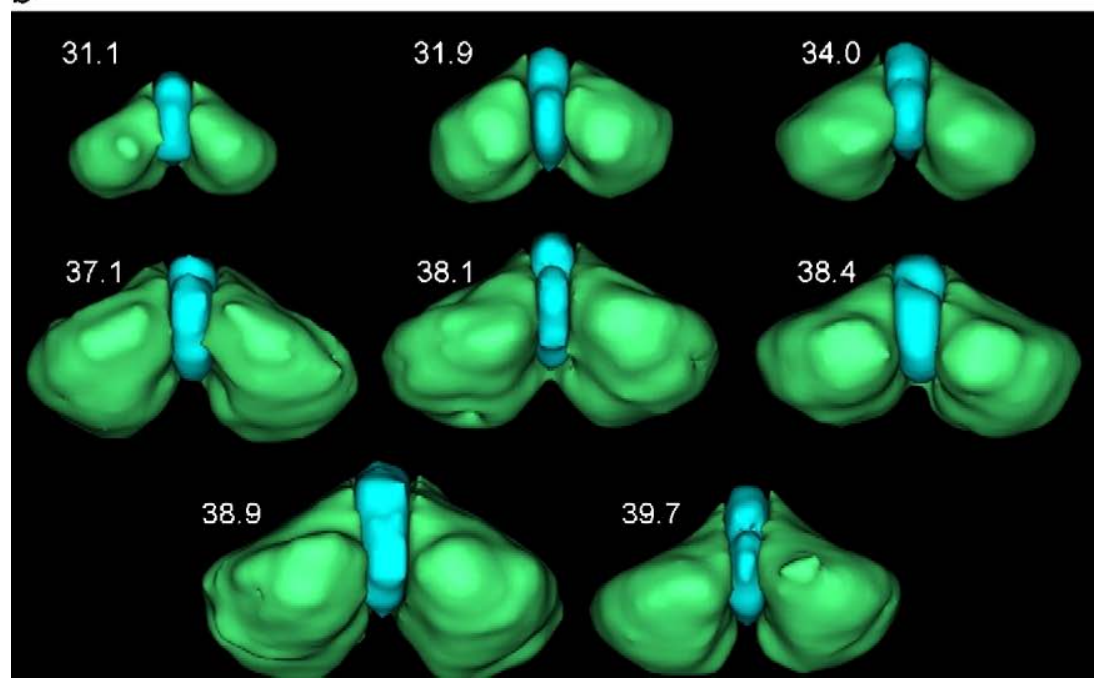

Fig. 6. Surface renderings. (a) Lateral view of cerebral brain exterior at different corrected GA shown in weeks in the upper right corner of each image. (b) Posterior view of cerebellar and vermis exterior at different corrected GA shown in weeks in the upper right corner of each image.

Using a logistic regression function, Kretschmann also described hippocampal growth using fixed specimens and these volumes are $31 \% \pm 12 \%$ larger (Kretschmann et al., 1986a,b) than our calculations. There are many possible sources for the significantly larger volumes obtained in the Koop and Kretschmann estimates compared to both our and McLennan's estimates. McLennan used fresh specimens, and we used in vivo data, whereas volume calculations for Koop and Kretschmann were from fixed specimen, and the hippocampal volume estimates required slicing. The fixation methods are not well documented, and therefore the relative effects of formaldehyde (which increase brain volume) compared to alcohol dehydration and celloidin or paraffin embedding (which decrease brain volume) is difficult to predict. For the hippocampal volume estimates, distortion from slicing can occur, and the effect of fixation on the hippocampus is totally unknown. Other possible sources of discrepancy are the much smaller number of brains and the inclusion of only male specimens in the Koop study. In Koop's discussion, he commented that their results were up to $150 \%$ higher than other estimates.

Other studies have used MR imaging data to determine brain volume in the neonatal time period (Huppi et al., 1998; Inder et al., 2005; Peterson et al., 2003). Huppi et al. and Inder et al. used dual echo (PD and T2) spin echo images co-registered to volumetric T1 images. An automated segmentation was performed based on signal intensity using a set of prototype tissue values previously selected by an expert operator. Using this technique, the intracranial contents were segmented into CSF, cortical gray matter, subcortical gray matter, unmyelinated white matter and myelinated white matter. This automated technique has the advantage of enabling evaluation of a large number of studies due to the minimal involvement of the user. However, the disadvantage is that the accuracy is difficult to assess. On the one segmented coronal image provided in both manuscripts, 


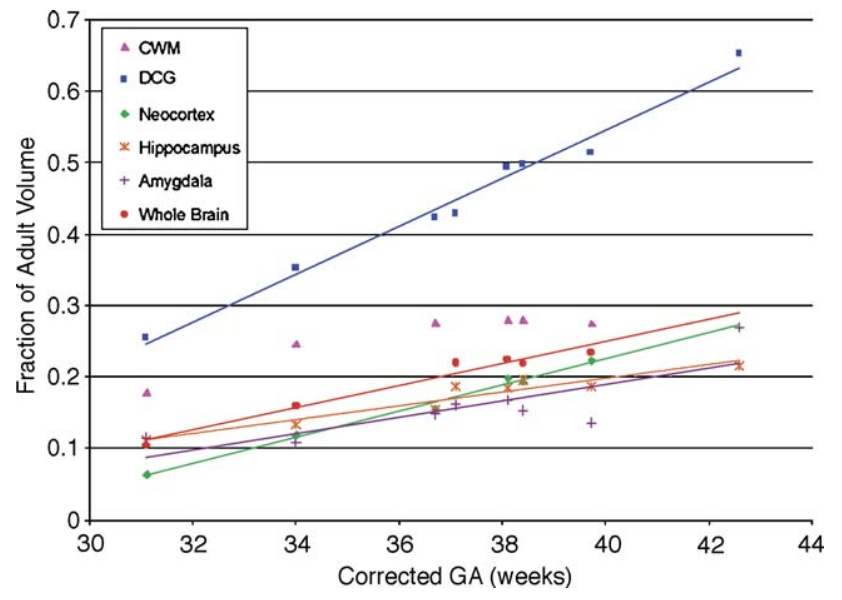

Fig. 7. Growth normalized by adult volumes. Volume of total DCG, CWM, neocortex, hippocampus and amygdala as well as whole brain versus corrected GA with volumes of each structure expressed as a fraction of the comparable adult volume reported by Filipek et al. (1994).

errors in tissue classification are evident. Huppi et al. performed segmentations into entire brain volume without ventricles, total gray matter and cortical gray matter that can be compared to our results. Our whole brain volumes are significantly smaller than those calculated by Huppi but had a similar growth rate (Fig. 4b). Our volumes are probably smaller since our detailed semiautomated segmentation does not include sulcal CSF which is included in semiautomated segmentations. Data on subcortical gray matter growth were not provided as they did not see any interval growth. This is quite different from our data where a growth rate of $1.5 \mathrm{ml} /$ week was identified $\left(P=3 \times 10^{-7}, r^{2}=\right.$ 0.98). Our ability to detect this small rate of growth may be due to the increased sensitivity of our detailed semiautomated technique.

Peterson used volumetric T2 FSE images (Peterson et al., 2003). A semiautomated segmentation method using a combination of thresholding and manual editing was used to segment the intracranial contents into cortical CSF, cortical gray matter, subcortical gray matter, white matter, ventricles, brainstem and cerebellum. This technique has the advantage of being faster than more detailed semiautomated techniques and more accurate than fully automated methods. Although editing can improve the results, thresholding can result in inaccurate segmentation of the gray-white junction where there is early myelination and inaccurate segmentation of the cortex from cortical CSF where there is increased gyral folding due to volume averaging effects. Typically this results in elevated cortical volume estimates. The figure provided in this manuscript shows overestimation of cortical thickness in the right perisylvian region as well as inclusion of the genu of the corpus callosum and posterior hippocampal bodies in the cortical gray matter volume. This study was focused on correlations between volumetric measures and neurodevelopmental measures. Scatter plots of total cerebral gray matter and total cerebral white matter to pontomedullary junction versus corrected GA are provided. These total gray matter volumes appear to be approximately $25 \mathrm{ml}$ larger and total white matter volumes appear to be as much as $40 \mathrm{ml}$ larger than our estimates.

With no gold standard, it is difficult to determine which method is the most accurate but in this study, accuracy of all segmented regions was visually confirmed by an experienced reviewer (PEG), and the high reliability measures ensured that the segmenter (MN) selected the same region on two different runs. In addition, it is reassuring that our data are concordant with the largest normative postmortem anatomic study to date. Therefore, we believe this technique is the most accurate. The fact that our technique detected volumetric changes where others have not suggests that our technique is more sensitive to small or regionally restrictive volumetric changes. In summary, we believe that the advantages of this technique are the accuracy of the volumes reported and the sensitivity to detect small volumetric changes. The disadvantage is the time required to segment each brain (approximately 7 days) which is impractical for large numbers of data sets.

Semiautomated techniques are useful in the analysis of large patient groups to assess for volume differences between large populations. However, even if the resulting volume differences are statistically significant and reproducible, one must be cautious in interpreting the findings as the accuracy of the segmentation has not been validated. In contrast, our detailed semiautomated technique is not appropriate for the evaluation of a large number of subjects because it is extremely laborious, but the segmentation has been confirmed by detailed visual inspection of each case by an experienced reviewer. Therefore, volumetric differences detected are likely to be accurate. In addition, volumes of small deep structures can be assessed. The ability to segment small structures such as the hippocampus or those that are greatly complex in form such as the neocortex with excellent $R^{2}$ values for the expected linear growth despite small numbers suggests that smaller numbers of patients may be required to distinguish normal from abnormal volumes and smaller deviations from normal may be detected. The ideal situation would be to combine the best from both of these methods and use the detailed semiautomated segmentation studies as gold standards for developing faster yet accurate semiautomated techniques.

Although these infants provided our normative data, they were scanned for clinical concerns and therefore are not completely normal. This is true in the vast majority of "normal" neonatal MR and pathological studies. The fact that the data behaved well in a coherent linear model with very favorable statistics, including $R^{2}$, suggests that our normative assumption is appropriate for structural analysis. Therefore, these normative neonates are likely to provide a good representation of the normal patterns of brain growth but truly normal neonates would reduce the "noise" coming from subtle abnormalities included in the normative subjects and improve the accuracy of the data base.

In summary, we have used a knowledge-based, reproducible and highly detailed semiautomated procedure to segment high resolution volumetric T1-weighted MR studies of neonatal brains. The resulting data provide the most detailed regional growth curves to date for the immature brain between 31 and 43 weeks corrected gestational age. This study provides further evidence of the heterochronous nature of cerebral development and the coordinated pattern of cerebral development. This study also provides initial estimates of regional brain growth and therefore regional vulnerability in the perinatal time period.

\section{Acknowledgments}

This work was supported by the William Randolph Hearst Fund (MN), K23 NS42758 (PEG) and in part by NS34189 (as part of the 
Human Brain Project) and the National Center for Research Resources (P41RR14075) and the Mental Illness and Neuroscience Discovery (MIND) Institute.

\section{References}

Bourgeois, J.P., Rakic, P., 1993. Changes of synaptic density in the primary visual cortex of the macaque monkey from fetal to adult stage. J. Neurosci. 13 (7), 2801-2820.

Castellanos, F.X., Lee, P.P., et al., 2002. Developmental trajectories of brain volume abnormalities in children and adolescents with attentiondeficit/hyperactivity disorder. JAMA 288 (14), 1740-1748.

Caviness Jr., V.S., Filipek, P.A., et al., 1989. Magnetic resonance technology in human brain science: blueprint for a program based upon morphometry. Brain Dev. 11 (1), 1-13.

Caviness Jr., V.S., Kennedy, D.N., et al., 1996a. The human brain age 7-11 years: a volumetric analysis based on magnetic resonance images. Cereb. Cortex 6 (5), 726-736.

Caviness, V.S., Kennedy, D.N., et al., 1996b. The developing human brain: a morphometric profile. In: Thatcher, R.W., Lyon, G.R., Rumsey, J., Krasnegor, N. (Eds.), Developmental Neuroimaging: Mapping the Development of Brain and Behavior. Academic Press, New York, pp. 3-14.

Caviness, V.S., Makris, N., et al., 1996c. MRI-based parcellation of human neocortex: an anatomically specified method with estimate of reliability. J. Cogn. Neurosci. 8, 566-588.

Filipek, P.A., Kennedy, D.N., et al., 1989. Magnetic resonance imagingbased brain morphometry: development and application to normal subjects. Ann. Neurol. 25 (1), 61-67.

Filipek, P.A., Kennedy, D.N., et al., 1991. Volumetric analyses of central nervous system neoplasm based on MRI. Pediatr. Neurol. 7 (5), 347-351

Filipek, P.A., Kennedy, D.N., et al., 1992. Neuroimaging in child neuropsychology. In: Boller, F., Grafman, J. (Eds.), Handb. Neuropsychol., 6. Elsevier, Amsterdam, pp. 301-329.

Filipek, P.A., Richelme, C., et al., 1994. The young adult human brain: an MRI-based morphometric analysis. Cereb. Cortex 4 (4), 344-360.

Filipek, P.A., Semrud-Clikeman, M., et al., 1997. Volumetric MRI analysis comparing subjects having attention-deficit hyperactivity disorder with normal controls. Neurology 48 (3), 589-601.

Fischl, B., Salat, D.H., et al., 2002. Whole brain segmentation: automated labeling of neuroanatomical structures in the human brain. Neuron 33 (3), 341-355.

Gogtay, N., Giedd, J.N., et al., 2004. Dynamic mapping of human cortical development during childhood through early adulthood. Proc. Natl. Acad. Sci. U. S. A. 101 (21), 8174-8179.

Hack, M., Breslau, N., 1986. Very low birth weight infants: effects of brain growth during infancy on intelligence quotient at 3 years of age. Pediatrics 77 (2), 196-202.

Hack, M., Breslau, N., et al., 1989. Differential effects of intrauterine and postnatal brain growth failure in infants of very low birth weight. Am. J. Dis. Child. 143 (1), $63-68$.

Hack, M., Breslau, N., et al., 1991. Effect of very low birth weight and subnormal head size on cognitive abilities at school age. N. Engl. J. Med. 325 (4), 231-237.

Huppi, P.S., Warfield, S., et al., 1998. Quantitative magnetic resonance imaging of brain development in premature and mature newborns. Ann. Neurol. 43 (2), 224-235.

Inder, T.E., Huppi, P.S., et al., 1999. Periventricular white matter injury in the premature infant is followed by reduced cerebral cortical gray matter volume at term. Ann. Neurol. 46 (5), 755-760.

Inder, T.E., Warfield, S.K., et al., 2005. Abnormal cerebral structure is present at term in premature infants. Pediatrics 115 (2), 286-294.

Kennedy, D.N., 1986. A system of three-dimensional analysis of magnetic resonance images. Department of Nuclear Engineering, Massachusetts Institute of Technology, Cambridge.

Kennedy, D.N., Nelson, A.C., 1987. Three-dimensional display from crosssectional tomographic images: an application to magnetic resonance imaging. IEEE Trans. Med. Imag. 6, 134-141.

Kennedy, D.N., Filipek, P.A., et al., 1989. Anatomic segmentation and volumetric analysis in nuclear magnetic resonance imaging. IEEE Trans. Med. Imag. 7, 1-7.

Kennedy, D., Meyer, J., et al., 1994. MRI-based topographic segmentation. In: Thatcher, W., Hallet, M., Zepffiro, T., John, R., Huerta, M. (Eds.), Functional Neuroimaging: Technical Foundations. Academic, New York, pp. 201-208.

Koop, M., Rilling, G., et al., 1986. Volumetric development of the fetal telencephalon, cerebral cortex, diencephalon, and rhombencephalon including the cerebellum in man. Bibl. Anat. (28), 53-78.

Kretschmann, H.J., Kammradt, G., et al., 1986a. Brain growth in man. Bibl. Anat. (28), 1-26.

Kretschmann, H.J., Kammradt, G., et al., 1986b. Growth of the hippocampal formation in man. Bibl. Anat. (28), 27-52.

LaMantia, A.S., Rakic, P., 1990. Axon overproduction and elimination in the corpus callosum of the developing rhesus monkey. J. Neurosci. 10 (7), 2156-2175.

LaMantia, A.S., Rakic, P., 1994. Axon overproduction and elimination in the anterior commissure of the developing rhesus monkey. J. Comp. Neurol. 340 (3), 328-336.

Makris, N., Meyer, J.W., et al., 1999. MRI-Based topographic parcellation of human cerebral white matter and nuclei II. Rationale and applications with systematics of cerebral connectivity. NeuroImage 9 (1), $18-45$.

McGraw, K.O., Wong, S.P., 1996. Forming inferences about some intraclass correlation coefficients. Psychol. Methods 4, 344-360.

McLennan, J.E., Gilles, F.H., et al., 1983. A model of growth of the human fetal brain. In: Gilles, F.H., Leviton, A., Dooling, E.C. (Eds.), The Developing Human Brain. John Wright, PSG Inc., Boston, MA, pp. $43-58$.

Meyer, J., Makris, N., et al., 1999. Parcellation of the human cerebral white matter: I. Technical Foundations. NeuroImage 9, 1-17.

Murphy, B.P., Inder, T.E., et al., 2001. Impaired cerebral cortical gray matter growth after treatment with dexamethasone for neonatal chronic lung disease. Pediatrics 107 (2), 217-221.

Pantelis, C., Velakoulis, D., et al., 2003. Neuroanatomical abnormalities before and after onset of psychosis: a cross-sectional and longitudinal MRI comparison. Lancet 361 (9354), 281-288.

Peterson, B.S., Anderson, A.W., et al., 2003. Regional brain volumes and their later neurodevelopmental correlates in term and preterm infants. Pediatrics 111 (5 Pt. 1), 939-948.

Rademacher, J., Galaburda, A.M., et al., 1992. Human cerebral cortex: localization, parcellation, and morphometry with magnetic resonance imaging. J. Cogn. Neurosci. 4, 352-374.

Rademacher, J., Caviness Jr., V.S., et al., 1993. Topographical variation of the human primary cortices: implications for neuroimaging, brain mapping, and neurobiology. Cereb. Cortex 3 (4), 313-329.

Shrout, P.E., Fleiss, J.L., 1979. Intraclass correlations: uses in assessing rater reliability. Psychol. Bull. 86, 420-428.

Sidman, R.L., Rakic, P., 1982. Development of the human central nervous system. In: Haymaker, W., Adams, R.D. (Eds.), Histology and Histopathology of the Nervous System. Charles C. Thomas, Springfield, pp. 3-145.

Sowell, E.R., Thompson, P.M., et al., 2004. Longitudinal mapping of cortical thickness and brain growth in normal children. J. Neurosci. 24 (38), 8223-8231.

Spencer, T.J., Biederman, J., et al., 2002. Overview and neurobiology of attention-deficit/hyperactivity disorder. J. Clin. Psychiatry 63 (Suppl. 12), 3-9.

Wilens, T.E., Biederman, J., et al., 2002. Patterns of psychopathology and dysfunction in clinically referred preschoolers. J. Dev. Behav. Pediatr. 23 (1 Suppl.), S31-S36. 